\title{
Nuevos aprendizajes. Ejemplos de lectura praxeológica de la Biblia
}

\author{
New learning examples of \\ praxeological reading of the bible
}

\section{Resumen}

El presente artículo es una propuesta de apropiación del movimiento teológico y pastoral que se ha fundamentado en el concepto de praxeología, orientado ya hacia la fundamentación metodológica y práctica de la lectura de la Biblia, proporcionando una reflexión sobre el uso y aporte de la Sagrada Escritura a todos los procesos generadores de fe y de transformación social y comunitaria.
En primer lugar se hará un breve esbozo de la situación actual de la exégesis, de su apertura a nuevos métodos y acercamientos a la lectura de la Biblia (conceptos que han sido profundizados por la Pontificia Comisión Bíblica en el documento: La Interpretación de la Biblia en la Iglesia, 1993). Luego se presentará la vertiente de la praxeología y sus contenidos metodológicos siguiendo los aportes de Carlos Juliao en su propuesta de legitimar el enfoque praxeológico para y desde la formación universitaria en Uniminuto, Colombia; y en tercer lugar, se aplicarán los conceptos, ideas y pasos de esta propuesta en la lectura de un texto bíblico del Antiguo Testamento.

Palabras clave: Biblia, Exégesis, praxeología, interpretación.

\begin{abstract}
Among the innumerable proposals and fields in the social, bumane and religious spheres, reflection on the use and contribution of the Holy Scriptures to all processes that generate faith and transformation plays a central role of articulation and explanation. The present article is a proposal for the appropriation of the theological and pastoral movement that has been founded on the concept of praxeology, now oriented to the methodological and practical foundation of reading the Bible.

First, it presents a brief review of the current situation of the exegesis, of its aperture to new methods and approaches to the reading of the Bible (concepts that have been deepened by the Pontifical Biblical Commission in the Church, 1993). Then it presents the current of praxeology and its methodological contents, following the contributions of Carlos Juliao in his proposal to legitimize the praxeological approach for and from university teaching in UNIMINUTO, Colombia, and third, the concepts, ideas and steps of the proposal will be applied to the reading of a Biblical text from the Old Testament.
\end{abstract}

Keywords: Bible. exegesis, praxeology, interpretation

\section{Recibido el 9 de agosto de 2012 y aprobado el 15 de agosto de 2012}

1 Licenciado en Sociales y Magister en Ciencias bíblicas y Arqueología-SBF Jerusalén. Docente de Sagrada Escritura de Uniminuto y Universidad de la Salle, Bogotá, Colombia. 


\section{Ubicación en el estado actual de la exégesis}

Los nuevos fenómenos que definen y orientan la sociedad y el mundo se rigen por criterios de pluralidad, diversidad, alternatividad, búsqueda o propuestas de identidad, etc. Estas realidades, también se perciben dentro de los procesos de fe que vive la Iglesia y los creyentes que intentan apropiar el aporte de otras disciplinas diferentes a la teología y la filosofía para dar respuesta a los retos que las nuevas sociedades les plantean tanto en el campo espiritual y religioso como en el humano y social. El sínodo de los obispos sobre la Nueva Evangelización, que se realizó en octubre de 2012, invita a abrir otros senderos, audaces, novedosos, diferentes que den respuesta a los caminos ya andados y que en esta época y contextos resultan insuficientes para proponer, articular, formar y madurar la fe. La exégesis bíblica está en estos procesos de reapropiación.

El estado actual de la exégesis bíblica plantea serios desafíos sobre la metodología empleada para obtener sus resultados; principalmente se presenta una ruptura en relación con el método clásico histórico-crítico. Al respecto, la Pontificia Comisión Bíblica en su aporte a la interpretación de la Biblia en la Iglesia comenta:

El método histórico-crítico se encuentra actualmente, en algunos ambientes, en competencia con métodos que insisten en una comprensión sincrónica de los textos, ya se trate de su lenguaje, de su composición, de su trama narrativa o de su esfuer- zo de persuasión. Por lo demás, al cuidado que tienen los métodos diacrónicos de reconstituir el pasado, se sustituye, frecuentemente, una tendencia a interrogar los textos situándolos en las perspectivas filosóficas, psicoanalíticas, sociológicas, políticas, etc., del tiempo presente. Este pluralismo de métodos y acercamientos es apreciado por unos como un índice de riqueza, pero a otros les da la impresión de una gran confusión (Pontificia Comisión Bíblica, 1993, p.27).

Para quienes se aproximan con rigor a los textos bíblicos, el aporte del método históricocrítico ha sido loable; no se trata, por simple antipatía o desinformación, de descartar un método, ni por la novedad de aplicar otro diferente; el mismo documento de la Comisión bíblica previene al respecto:

En consecuencia, se considera necesario sustituir el paciente trabajo de la exégesis científica con acercamientos más simples, como tal o cual práctica de lectura sincrónica, que se considera suficiente; o inclusive, renunciando a todo estudio, se favorece una lectura de la Biblia llamada "espiritual". Con este término se entiende una lectura guiada únicamente por la inspiración personal subjetiva y destinada a nutrir esta inspiración. Algunos buscan en la Biblia sobre todo el Cristo de su visión personal y la satisfacción de su religiosidad espontánea. Otros pretenden encontrar en ella respuestas directas a todo tipo de cuestiones personales o colectivas. Numerosas sectas proponen como única interpretación verdadera aquella de la cual afirman haber tenido la revelación (Pontificia Comisión Bíblica, 1993, p.28). 
Se trata de procurar aproximarse al texto con todas las herramientas posibles y de establecer canales de comprensión, interpretación y actualización entre el texto, los lectores - pensados como comunidad-, y la realidad que los envuelve.

En este sentido, J. S. Croatto ayuda a precisar el aporte de los métodos diacrónicos al afirmar que: "toda lectura es hermenéutica y debe pasar por el texto; pero también es cierto que todo texto, aunque analizado exhaustivamente y casi 'clausurado' por la exégesis histórico-crítica (paso necesario) permanece 'polisémico' en su lectura concreta e históricamente situada" (Croatto, 1987).

Al hablar de la presentación exegética de un texto bíblico, de inmediato el lector se conecta con un método y espera escuchar algunas novedades, siguiendo el caso de Pablo en el areópago ateniense (Hch 17, 16-21).

Pero es importante tener en cuenta que no se trata de ofrecer respuestas doctas ni explicaciones contundentes, sino de mostrar un camino, asumiendo tres factores: el texto, con todas sus características literarias y trasfondos históricos, socio-culturales y religiosos; la persona que lo lee/estudia/ora, con sus "intencionalidades" y vacíos, sus expectativas y necesidades, sus intimidades e influjos contextuales; y las motivaciones con/por las que se acerca al mismo, de conocimiento, comprensión, respuestas o meditación.

Otro inconsciente que se maneja al hablar de exégesis es el de los contenidos, los cuales son relacionados con tal o cual teoría literaria, arqueológica, doctrinal o línea de estudios bíblicos, bien sea diacrónica, sincrónica, hermenéutica, que cada vez están más bifurcadas, y a partir de este filtro se juzgan o emiten juicios personales de valor. Es cierto que se debe mostrar (y demostrar) los resultados de toda investigación bíblica que el biblista (estudioso de la Biblia) emprenda, y por esta razón encontramos cantidad de libros y artículos de revistas en las secciones de novedades de las librerías especializadas; pero, ¿los lectores se han detenido a pensar en el proceso de gestación y trabajo de parto que ha asumido el biblista para poder ofrecer al público lector un producto que infortunadamente puede reducirse en la crítica como algo bueno, malo, repetitivo, corto, interesante, insuficiente, etc.? Aunque este es el ejercicio cotidiano de todo escritor, en este caso se trata del testimonio de fe de un creyente que escribe para creyentes.

Consiste, entonces, en abrir los horizontes interpretativos de los textos sagrados, seguros de encontrar en ellos esa fuente inagotable de agua viva $(\mathrm{Jn} 4,10)$. Estos horizontes no son solamente puntos de vista o resultados, sino el efecto de la lectura atenta y creyente de la Biblia, que en sí misma proporciona un camino a seguir y brinda las herramientas suficientes de comprensión, profundización y aplicación de los textos; no es cuestión de "mirar" sino de "salir al encuentro", como lo hizo el padre de aquel hijo pródigo (Lc 15, 20), y como lo experimentó Pablo en su propio camino vocacional, en su experiencia de encuentro con Cristo resucitado (Flp 3, 12-16), culminando con esa magnífica expresión que invita a no conformarse con lo adquirido: "desde el punto a donde hayamos llegado, sigamos en la misma dirección" (NBJ, p.1636).

Como son procesos humanizadores y de fe, es relevante que quien escribe, enseña y predica desde la Biblia se arriesgue a exponer no solamente los resultados de lo que con ahínco y gusto se propone producir, argumentar, exponer, sino, también, a compartir la experiencia de construcción que implica brindar resultados concretos, pero ante todo requiere el constante encuentro con el texto, los presupuestos previos a esos encuentros, las múltiples motivaciones y situaciones con que se produjeron en ellos, la vivencia de fe, sus "desiertos" espirituales, etc.;

2 Sobre esta cuestión ver J.S. Croatto, Biblical Hermeneutics (1987), 36ss; del mismo autor, "L'herméneutique biblique en face des méthodes critiques: défi et perspectives", Vetus Testamentum, supl. 36 (1985): 77ss. 
todo un gozoso trabajo de parto, que conduce a la plenitud de haber asumido un texto para sí y sus lectores, para su crecimiento y el de las comunidades que puedan apropiar sus esmerados aportes y enriquecerse con sus enseñanzas.

\section{Definición de praxeología}

Antes de entrar en contacto con el texto bíblico, es necesario trazar el camino metodológico que a través de este artículo se propone a los lectores. En primer lugar habrá una aproximación al concepto de praxeología y luego la aplicación metodológica en el texto bíblico.

¿Qué se entiende por praxeología? El origen y aplicación del concepto se cree que surgió en Francia a finales del siglo pasado, bajo la pluma de L. Bourdeau (1882), a partir de una exigencia: reconocer, contra la clasificación de Auguste Comte, una "ciencia integral" que coordine todas las otras y de las cuales se trataría de rehacer la unidad. Quince años más tarde, A. Espinas (1897), utilizó este vocablo para designar a una "ciencia general", "con las formas más universales y los principios más elevados de la acción en el conjunto de los seres vivos capaces de moverse"3. El término de praxeología se vuelve a encontrar, más adelante, en estudios económicos, en un periodo que se extiende de 1926 a los años cincuenta, primero en la URSS (Sluckil, 1926), luego en Australia (Mises, 1983), y sobre todo en Polonia, donde T. Kotarbinski, en su Tratado del buen trabajo, la define como "teoría general de la acción eficaz" (Lodz, 1955). Estas definiciones se aplicaron a los campos de la Economía, sociología del trabajo, ciencias de la organización y de la decisión, entre otras (Juliao, 2011, p. 13).

Siguiendo el aporte y aplicación de Carlos Juliao, quien no se apresura a dar una definición de esta "ciencia" o "praxis", pero remite a la experiencia discipular (Jn 1, 38-39) en la que lo importante no son los resultados cognitivos sino el hecho mismo de estar en camino, el "ir" ercomai, "ver" oraw y "quedarse" o permanecer en la experiencia, menw (Brown, 1999, p. 288). Este autor parte de tres presupuestos: El primero, como punto de partida es antropológico, donde "la persona humana es un ser praxeológico, es decir, un individuo que actúa (¿actante?), que reflexiona sobre su actuar, que busca mejorar sus acciones y, en últimas, ser feliz" (Juliao, 2011, p. 22). El segundo presupuesto que plantea este autor es el de la praxeología como un "discurso sobre la acción”, el logos de la praxis (de ahí el concepto), siendo así:

más consciente de su lenguaje, de su funcionamiento y de lo que en ella está en juego, sobre todo del proceso social en el cual el actor o practicante está implicado y del proyecto de intervención que construye para cualificar dicho proceso; todo esto con el fin de acrecentar su pertinencia y su eficacia liberadora (Juliao, 2011, p. 27).

El tercer presupuesto que fundamenta este enfoque es el de la "praxeología como proceso"; según Juliao, la praxeología: "es la construcción de saberes de la acción (lógica científica)" (2011, p. 35), que busca, por lo tanto, elaborar, experimentar y validar modelos de acción, útiles para la gestión de la praxis. Es en este presupuesto donde se presenta la metodología praxeológica como una propuesta metodológica, con sus cua-

3 Esta cita textual y la idea de construcción sobre el concepto de praxeología, lo articula Daniel Scheinsohn, en su obra titulada El poder y la acción (2011, p.19).

4 Es de notar que no se trata solamente de una facultad humana sino de la capacidad de percepción profunda y subsiguiente la fe, asociándose así con el verbo creer. Creer posibilita ver en profundidad (Ramos Pérez, 2004). Cfr. (Sánchez Castelblanco, 2009, p. 251).

5 "El hombre desea estar con Dios; trata constantemente de superar la temporalidad, el cambio y la muerte, en busca siempre de algo permanente. Jesús responde con el desafío total de la fe: $<<$ Venid y lo veréis $>>$. A lo largo de todo el evangelio de Juan se recurrirá al tema de $<<$ venir $>>$ a Jesús para describir la fe. También $<<$ ver $>>$ a Jesús con discernimiento es otra forma de describir la fe en Juan" (Brown, 1999, p. 294). 
tro fases del ver, juzgar, actuar y de devolución creativa, guiadas por cuatro preguntas fundamentales: ¿Qué sucede? ¿Qué puede hacerse? ¿Qué se hace en concreto? y ¿qué aprendemos de lo que hacemos?

Al hacer referencia a la interpretación bíblica, acuñada con el término hermenéutica, se perciben dificultades de comprensión y de aplicación del concepto y sus procedimientos, que, partiendo de los presupuestos y la metodología praxeológica, invitan a los lectores a situarse desde el punto de vista del lector-actorhermeneuta, sujeto de la praxis bíblica. El consenso de autores indica que hermenéutica no implica solamente el ejercicio de interpretación de los textos bíblicos, "sino sobre todo de la actualización del mensaje contenido en ellos, de manera que se ponga de manifiesto su relevancia para el tiempo presente y para las situaciones que les toca vivir a los hombres de hoy" (Levorati, 1977, p. 302). Por ello es valioso el concepto de "acción" con sus connotaciones de pluralidad, imprevisibilidad y fragilidad; los textos bíblicos recogen la pluralidad de expresiones y sentires propios del pueblo de la Biblia, tanto en el Antiguo como en el Nuevo Testamento, referido a la fundamentación del judaísmo y del cristianismo respectivamente ${ }^{6}$; se habla entonces de tradiciones orales y escritas, corrientes de pensamiento, escuelas, influjos culturales y literarios, teologías, etc., para explicar la diversidad textual que encontramos en las páginas de la Biblia. La exégesis históricocrítica ha procurado señalar estas divergencias y explicarlas a partir de las etapas redaccionales de los libros, pero, en el plano humano, estamos ante el aporte de las comunidades, centralizadas o periféricas, que apropiaron los textos y los aplicaron a su propia realidad envolvente, en cada época y situación histórica que vivieron. Se traza, por tanto, una tarea praxeológica en los mismos textos bíblicos.

Tomando como punto de apoyo el principio de la "teoría de la acción", es necesario recordar el aporte de la Pontifica Comisión: Hay "una tendencia que preconiza una lectura creyente, pero libre, espontánea, personal y pneumática de los textos bíblicos, cuestionando radicalmente la búsqueda de sentido literal y la validez de la comprensión histórico-crítica de la escritura" (Levorati, 1977, p. 302), considerando el pasado bíblico, como pasado, irrelevante, para incluir una actitud creyente que busca en la Biblia el encuentro actual con Dios; "es preciso aceptar el texto como acontecimiento" (Levorati, 1977, p. 303), con pluralidad de sentidos, no como letra muerta y concluida. Un texto no existe en si como "letra muerta" sino a la espera del lector que le confiere vida e inmortalidad.

Este acto es un fenómeno complejo de encuentro y de interacción entre el mundo del texto y del lector. No es inmediato y natural debido a la distancia cultural e histórica entre estos mundos. Así resulta más importante interpretar que comprender (ejercicio cognitivo, mental y sensitivo). La interrelación implica un amplio movimiento desde la comprehensión, apoyada de la expiación, hacia la cuestión del sentido, hasta la apropiación, o una aplicación, personal por parte de los lectores.

El siglo XX ha visto un interés creciente por la hermenéutica como teoría general y campo de investigación de la interpretación; se interesa por los procesos y el acto de comprender, las

6 En este sentido, son reconocidos los aportes de varios exégetas que proponen una pluralidad de teologías en la biblia.A continuación se presenta una reseña bibliográfica a partir de la investigación del grupo "Teología, Biblia y Religión", registrado en Colciencias (col 0081915): "Historia y método de la Teología Bíblica", que desarrolla el proyecto de investigación "Las teologías del sacrificio en el Antiguo Testamento", presentado por la Facultad de Teología y aprobado por la Dirección de Investigaciones de la Universidad de San Buenaventura, Bogotá. Los artículos pertenecen a diferentes ámbitos académicos de estudios de la Teología Bíblica y fueron considerados en su orden: Bonora (1988); Lemke (1992); Morgan (1992); Brueggemann (2007); Segalla (2006). Asimismo se consideraron como fuente otros textos que abordan la Teología Bíblica: Kraus (1979); Goppelt (1982); Segalla (1994); Eichrodt (1975); Von Rad (1982). 
mediaciones de la comprensión humana como el lenguaje, la estructura de comunicación, los métodos y sus efectos (Parmentier, 2004, p. 18).

Sin cerrar definitivamente la discusión, sino brindando aportes para enriquecerla, se propone en las páginas siguientes aplicar el enfoque praxeológico, con su metodología propia, en la Biblia, asumiendo los sentidos de pluralidad, de imprevisibilidad y de fragilidad, propios de los autores de los textos bíblicos y de los lectores que entran en contacto con sus páginas, con sus cargas emocionales, sus sentires personales y comunitarios, sus expectativas de formación, apropiación y espiritualidad a partir del encuentro.

\section{Aplicación de la praxeología en la lectura de la Biblia: ¿Es sabio Balaam o su burra? (estar dispuestos a dejar que una burra nos hable)}

A continuación se propone la lectura de un texto del Antiguo Testamento, Números 22, 21-35, tal vez "inconsciente", por ser uno de esos textos que son poco estudiados o aplicados en la enseñanza y demás actividades formativas en el campo de estudios bíblicos, de formación religiosa y catequética, y que puede servir de ejemplo para realizar otras búsquedas de sentido de las cuales se ha hablado y con seguridad todo biblista y creyente busca, sin separarse del texto, pero sin ser tampoco ajenos a la propia realidad y a la experiencia de otros caminantes.

La historia de Balaám invita a los lectores a descubrir y profundizar uno de los textos bíblicos que se presentan redactados con géneros literarios poco comunes y que revisten tonos anecdóticos, populares y pintorescos a comprender que los escenarios de aprendizaje son diversos, pero no ajenos a nosotros, e incluso parten de cada uno. El ejercicio consiste en buscar lógicas diferentes que se presentan en el texto y seguir la metodología praxeológi- ca para lograr su comprensión y actualización a nivel personal, comunitario, investigativo, etc., en lo referente a la praxis de vida y de la acción. A la vez será propuesto como una didáctica para desarrollar cada acción con sentido praxeológico.

\section{A. Ver}

\section{Se levantó Balaán muy temprano, preparó su burra, y se fue con los jefes de Moab.}

Nм 22,21.

¿En qué punto estamos ubicados? Es necesario e importante tomar conciencia del momento que cada uno está viviendo, a niveles personal, familiar, social y comunitario, incluidos los procesos de aprendizaje, de encuentro y de compartir bíblicos. El relato invita al lector a tomar el puesto de Balaám. Los textos bíblicos no solamente presentan situaciones vividas por personajes que aparecen como reales en los mismos relatos, sino que toman posiciones personales en relación con sus mismas situaciones. A partir del análisis praxeológico, muchas de las prácticas son autómatas, inconscientes, superficiales, poco reflexivas. Partiendo de la pregunta ¿qué sucede?, se pueden precisar las situaciones que se están viviendo, las que se señalan en el texto y las que de la vida cotidiana se pueden describir. En este punto hay un acercamiento al método narrativo que habla de la "puesta en escena", en la que el enunciado narrativo, como lectores, está ante los ojos (Parmentier, 2004, p. 188) y que como tales se invita a percibir, recorriendo un campo de experiencia (Simian-Yofre, 2001, p. 150), hasta llegar a comprender el itinerario que el texto propone. ¿Balaám se levantó de madrugada y preparó su burra de manera espontánea e intuitiva o lo hizo de una manera pensada y controlada? Está presente una acción que es necesaria comprender, explicar si responde a objetivos o a intuiciones o estímulos espontáneos. La práctica humana se ve reflejada en esta expresión popular: "Al que madruga Dios le ayuda"; el fenómeno más común de las personas es el de 
levantarse a cumplir con sus proyectos diarios, a desempeñar sus funciones familiares, a ejercitar sus labores sociales y económicas, etc., pero, ¿lo realizan conscientemente?

Si se lee el texto en su contexto inmediato, el v. 20 indica cuál es la motivación profunda de esta acción inicial: Dios mismo se acercó a Balaám y le indicó qué debía hacer. No se trata, entonces, de una práctica espontánea e intuitiva, sino de una praxis elaborada y reflexionada; ante esta evidencia, ¿demostrará Balaám con hechos y evidencias que ha comprendido esta "voz de Dios" y lo aplicará en los hechos que van a sucederse (se trata de un saber-hacer demostrado)?

\section{B. Juzgar}

Al verlo ir, se encendió la cólera de Dios, y el ángel del Señor se puso delante de él en el camino para cerrarle el paso. Balaám iba montado en su burra y lo acompañaban dos de sus criados. La burra, al ver al ángel del Señor parado en el camino, con la espada desenvainada en la mano, se desvió del camino y se fue por el campo. Balaám le daba golpes para hacer que regresara al camino. El ángel del Señor se interpuso en un lugar estrecho del camino, en medio de las viñas, con pared a un lado y a otro. La burra, al ver al ángel del Señor, se fue contra la pared apretando contra ella el pié de Balaám, que se puso a apalearla de nuevo. Una vez más el ángel del Señor se les adelantó parándose en un paso muy estrecho, sin desviación posible ni a un lado ni a otro. Cuando la burra vio al ángel del Señor, se tumbó con Balaán encima, mientras él, enfurecido, le pegaba con la vara (Nm 22, 22-27).

Para este segundo paso se toma como referencia la pregunta ¿qué puede hacerse? Esta fase se despliega como una etapa hermenéutica, donde se encuentran otras formas de enfocar la problemática de la práctica; la tarea en este momento es la de visualizar y juzgar para comprender la práctica; así se llega a conformar el propio punto de vista y se aúnan esfuerzos para elaborarlo (Juliao, 2011, p. 38). Es también una fase de reacción. El texto proporciona una amplia gama de descriptores y acontecimientos que permiten una mayor pluralidad de sentidos del mismo; los personajes que sobresalen son el ángel del Señor y la burra de Balaám, ya que es la vidente de Dios; los dos acompañantes, que son jóvenes, no asumen un rol dentro de la escena, lo cual no indica que no lo tengan efectivamente o que más adelante proporcionen un aprendizaje, no hay palabras vanas en la Biblia. Los tres momentos que desarrolla este segmento indican tres circunstancias que fuerzan a tomar tres opciones: en el primero, es posible desviarse del camino, dejando de lado un derrotero propio y en cierto modo huyendo de la misma realidad; el segundo hace ver la necesidad de afectarse ante procesos de fe, de madurez, de consolidación personal; el ver la realidad implica asumirla con fricciones, rupturas, complejidades, perplejidades; la persona misma es objeto de aprendizaje, no solamente es el sujeto de lo que aprende; en este sentido, él está tomando conciencia de su proceso y no puede definir ni identificar totalmente los resultados: así como el texto muestra otras posibilidades, el ser humano se abre a la pluralidad de situaciones con sus sentidos implícitos.

En relación con la primera fase, el contexto pone en evidencia una ruptura fundamental que el análisis hace ver como incoherente: si Dios envió a Balaám, ¿por qué ahora se enfurece, se pone delante del camino y procura cerrar el paso al profeta? El análisis literario indica que esta contradicción puede corresponder a una tradición diferente, como la Elohista, ya que emplea la expresión "el ángel de Yhwh", y no Dios mismo, acentuando así la distancia entre el hombre y Dios al poner un mediador como interlocutor. Luego, la burra de Balaám percibe esta presencia y reacciona esquivando los ataques del mensajero de Dios. Balaám debe juzgar estas dos situaciones, evaluarlas y formalizar paradigmas de su praxis; es decir, no se trata solamente de la reacción de un animal, sino de analizar el por qué y captar a profundidad su significado; es la comprensión de la que trata la fase, generar un modelo de acción que responda a la situación concreta y enriquezca el potencial del saber-hacer (Juliao, 2011, p. 39). Pedro, haciendo una aplicación de este pasaje del Antiguo Testamento (2Pe 2, 15-16), señala que quienes se 
desvían del camino recto se convierten en seguidores de Balaám y advierte cómo, ante su insensatez, este profeta fue corregido por su asno.

Balaám ha de comprender la magnitud de lo que el rey de Moab, Balac, está a punto de pedirle: maldecir al pueblo de Dios, lo cual lo pone en una condición de enemigo del mismo Dios; no basta solamente haber escuchado la voz divina y respondido a ella, sino que se trata de responder con criterio a ese llamado y entrar en un diálogo coherente de fe. Muchas veces las personas creen que siguen a Dios cuando atacan a otras personas porque no se ajustan a los parámetros oficiales o morales que la sociedad creyente ha dictaminado; otros creen que no hay valores cristianos en quienes asumen modas o formas de vida diferentes, especialmente hablando de los jóvenes y las nuevas generaciones; si Dios intenta detener al profeta es para que él mismo tome conciencia de su camino y de su destino, de lo que va construyendo y de las consecuencias de ese obrar, de esa praxis de vida.

El segundo aprendizaje es el de la burra que se desvía del camino; no se trata de un simple caso de terquedad del animal; aquí hay todo un paradigma opuesto al tradicional: se puede aprehender de lo que, en apariencia, es insignificante o no proporciona ningún conocimiento ni aporte relevante. Es muy fácil seguir los paradigmas establecidos, los más cortos, los menos complejos y de resultados inmediatistas, sin percibir que la vida es más compleja, variada, rica de sentidos. Juliao habla de cuatro momentos dinamizadores: el de la problematización de la propia observación, la formulación de una hipótesis de sentido, la formulación de los discursos y el retorno a las fuentes (2011, p. 39). Hasta ahora, en el caso de Balaám y su burra, el profeta no ha desarrollado ninguno de estos momentos, sino que se limita a golpear brutalmente a su animal para proseguir con su inútil caminar. Preguntas y pautas orientadoras:

En este camino de vida, ¿es preciso reconocer algunas limitaciones o vacíos?

$¿$ ¿Hay elementos externos que posibilitan/ frenan el propio camino?
$¿$ Se ha aprendido a seguir nuevos caminos o existe la tendencia a conservar esquemas fijos de circunstancias pasadas?

Es necesario armar nuevas coherencias a partir de nuevas circunstancias.

El texto ofrece una precisa aplicación y resolución de esta fase, ya que presenta de una manera pintoresca, por medio de una fábula, la toma de conciencia de Balaám y la consecuente intervención de Dios:

Entonces el Señor abrió la boca de la burra, que dijo a Balaám: ¿qué te he hecho yo para que me pegues por tercera vez? Balaám respondió: Te burlas de mí. Si tuviera a mano una espada, ahora mismo te mataría. La burra dijo a Balaám: ¿no soy yo tu burra, que te he servido siempre de cabalgadura hasta hoy? ¿Te he hecho yo alguna vez cosa semejante? Respondió Balaám: no. El Señor abrió los ojos a Balaám, y éste vio al ángel del Señor en el camino con la espada desenvainada en la mano. Balaám se inclinó y se postró en tierra. El ángel del Señor le dijo: ¿por qué has pegado a tu burra por tres veces? Era yo quien te cerraba el paso, pues tu viaje no es de mi agrado. La burra me ha visto y por tres veces se ha apartado de mí. Gracias a que se ha apartado, que si no, habría sido yo quien te hubiera dado muerte a ti, dejándola a ella con vida. Balaám respondió al ángel del Señor: ¡He pecado! No sabía que eras tú quien me cerraba el paso. Si este viaje te desagrada, ahora mismo regreso a mi tierra (Nm 22, 28-33).

Balaám no era consciente de esta "verdad de Dios", su observación resultaba limitada y condicionada por su propia visión de los hechos; aún escuchando hablar a su burra que le reclamaba por su conducta incoherente seguía obstinado en su propio punto de vista, hasta que el mismo animal le hace tomar en cuenta que ha permanecido fiel a él, no como un accesorio más sino como una presencia significativa para él; en este momento es cuando él es capaz de comprender que urge otra acción a partir de su potencial creador e innovador. El discurso o marco teórico de esta narración se tipifica en la voz de Dios, quien le hace ver otra fuente de conocimiento desde la óptica de la burra y no desde su única perspectiva, con una inclusión que está expresa con el desagrado por el viaje de Balaám, como lo muestra 
el análisis literario en los vv. 22 y 32 . El retorno a las fuentes está expresado, en primer lugar, por el hecho de la postración de Balaám ante Dios y en segundo lugar, por las palabras que pronunció en las que replantea su camino. Es necesario ser rigurosos en la elección de fuentes y su análisis, hasta, incluso, reformular la propia problematización. Este es uno de los aspectos claves de toda lectura de la Biblia, el de comprender que no hay un solo sentido en el texto, sino un prisma de posibilidades y lecturas? ${ }^{7}$.

A partir de este segundo momento, se presentan algunas preguntas y pautas para continuar la reflexión:

¿Se han generado nuevos aprendizajes aún asumiendo aspectos que no son incluidos tradicionalmente en los procesos cognitivos o de madurez en la fe? Se trata de darle su lugar a la corporeidad, las emociones y relaciones, la espiritualidad entendida como sentido de la vida.

¿Hacia dónde nos proyectamos?

¿Qué esperamos de esta práctica/curso/proyecto/encuentro/catequesis/reunión, etc.?

"cada quien, con lo que es, con lo que sabe y con lo que tiene, hace lo que puede".

Es importante reconocer lo que ya se sabe y se tiene, presuponiendo que se quiere ser mejores.

\section{Actuar}

\section{El ángel del Señor le dijo: vete con esos hombres, pero di solamente lo que yo te mande. $Y$ Balaám siguió con los dignatarios de Balac}

Nm 22,34-35.

La pregunta clave de esta fase es ¿qué hacemos en concreto?, entendida como una etapa fundamentalmente programática (Juliao, 2011, p. 40). En el texto se encuentra de nuevo una inclusión, en los vv. 20 y 35, que indica un primer comienzo programático: "Levántate y vete [...] Pero has de cumplir la palabra que yo te diga" y otro nuevo comienzo, "vete $[. .$.$] pero no digas nada más$ que lo que yo te diga", ya que no se cierran en los hechos ni en la temporalidad. En el caso del primer envío, Balaám elabora una construcción a partir de la experiencia del caminar y de la reacción de su burra; para el segundo acto ya se trata de un continuar reflexivo, praxeológico, aplicado a su propia praxis; es aquí cuando se potencializa el papel de las/los siervas/os —otra traducción posible es jjóvenes! De la raíz $\rho[v$, quienes más que funcionarios o actores obligados son acompañantes del profeta: ellos se convierten en aprendices-practicantes . Este proceso de conocimiento (de sí mismo, de los diversos actores, del medio) lleva a la eficacia de la acción: Balaám comprende por qué y para qué va al encuentro con Moab, precisamente como mensajero de Yhwh y sabe cómo lo hará; ha trazado un proceso desde la experiencia y la experimentación, el análisis y la interpretación, y ha descubierto paradigmas de su praxis como profeta: comprende los procesos, identifica las problemáticas y determina las finalidades (Juliao, 2011, p. 41). Se trata, en suma, de una transformación real de la práctica y de su propia vida como practicante. Así, los lectores van llegando a la finalidad que se propone a partir del encuentro con los textos bíblicos: "se trata de desencadenar un verdadero proceso de cambio, de transformación: ¿cómo responder concretamente a las esperanzas, iniciar los desplazamientos, vivir lo novedoso e innovador?" (Juliao, 2011, p. 41).

Nuevamente se escucha la voz de Dios, es decir, de quien comprende las situaciones desde su perspectiva salvífica; la mediación ha sido eficaz, tanto de parte del mensajero de Yhwh como de la burra de Balaám. Por esto, Balaám

7 El documento de la Pontificia Comisión, en el capítulo I habla de "Métodos y acercamientos para la interpretación"; Barton propone, entre otros, lecturas literarias, enfoques postestructuralistas, lecturas políticas y la interpretación feminista, etc., y Parmentier presenta modelos como el kerigmático y el experiencial, además de los tradicionales histórico y narrativo.

Polisemia No. 14, 110 - 121. Nuevos aprendizajes. Ejemplos de lectura praxeológica de la Biblia". Bogotá, ISSN: 1900-4648. Julio - diciembre de 2012 
nuevamente guarda silencio, para asumir la actitud de escucha, propia del investigador, del practicante, del profesional, del praxeólogo, del creyente. Este efecto teológico-existencial lo expresa el texto recurriendo de nuevo al fenómeno

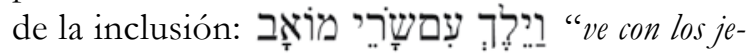
fes de Moab" (vv.21.35).

\section{Preguntas para la reflexión:}

¿De qué manera el lector se puede comprometer con los objetivos de este proceso de aprendizaje?

¿Cuál será su aporte al grupo/comunidad/ sector?

¿Cómo poner en práctica los conocimientos adquiridos?

\section{Devolución creativa}

Esta etapa considerada fundamentalmente prospectiva (Juliao, 2011, p. 43) responde a la pregunta ¿qué aprendemos de lo que hacemos? El pasaje en estudio cumple la función introductoria de toda la perícopa de Balaám, que se considera en los capítulos 22-24 del libro de los Números. Los cuestionamientos que pudieron surgir a la exégesis bíblica sobre por qué un profeta de Mesopotamia resulta bendiciendo a un pueblo como Israel, de camino por el desierto y con una imagen bastante negativa por parte de los pueblos que sufrían su devastador paso, se ve respondida por este pasaje ameno y de profundidad teológica; se traza un cambio en la visión profética; se muestran nuevas vías de acción por parte de Dios y se consolida un actuar marcado por los gestos que indican bendición y culto. Puede presentarse a la vez una ironía, ya que quienes han sido testigos del paso (pascua) liberador del Dios del pueblo de la Biblia, optan por maldecirlo y atacarlo, mientras que quien desconoce estos hechos históricos y venera otras divinidades, resulta ser quien lo reconozca. Encontramos, en este sentido, afinidades con el relato universalista de Jonás y el cambio de perspectiva teológica de la retribución replanteada en Job: Nadie puede oponerse ni luchar contra quienes Dios ha elegido y constituido depositarios de su bendición ya que sería luchar contra el mismo Dios. Se expresa de manera creativa el resultado de un proceso de aprendizaje y de conocimiento de Dios y del mismo hombre.

\section{Conclusión}

El acercamiento a la praxeología como método de lectura de los textos bíblicos se abre como una posibilidad de entrar en el proceso mismo de transmisión del texto como contenido de verdades de fe, valores religiosos y principios éticos y de vida, tanto personal como en comunidad. Pero también permite descubrir en las narraciones mismas una clave de lectura hermenéutica de los acontecimientos que como personas y sociedad nos inquietan y reclaman solución o por lo menos comprensión.

No se trata de inventar otro método más, sino de apropiar las herramientas de la exégesis bíblica (método histórico-crítico, narrativa, retórica, estructuralismo y otros posibles acercamientos) y articularlos con la hermenéutica, entendida como interpretación del texto y actualización del mismo en los contextos determinados de nuestra vida.

Pero no se queda solo en lograr mejores aproximaciones y nuevas lecturas de los textos, sino en generar procesos de reflexión activa sobre la praxis misma de las personas, entendidas como sujetos de su historia y como agentes de cambio para los demás. Por tanto, es un aporte para propiciar pautas de autoevaluación, de apropiación de mejores instrumentos y la consecuente generación de nuevos aportes tanto de saber como de praxis. Se trata de reconocer cómo se vive, a nivel existencial, en todas las dimensiones humanas, para desde ahí vivir mejor, construyendo un proyecto de vida desde la fe y brindando luces de transformación social siguiendo la experiencia bíblica. 


\section{Referencias bibliográficas}

Barton, J. (Ed.). (2001). La interpretación bíblica, hoy. Santander: Sal terrae.

Bourdeau, P. L. (1882). Théorie des sciences. Plan de science intégrale. Paris: Germer Baillière.

Brown, R. E. (1987). El Evangelio según Juan I-XII. Madrid: Cristiandad.

Brueggemann, W. (2007). Teología del Antiguo Testamento. Salamanca: Sígueme.

Bonora, A., \& Segalla, G. (1988). Teologia biblica. En P. Rossano, G. Ravasi \& A. Girlanda (Eds.), Nuovo dizionario di teologia biblica (pp. 1533-1552). Milano: Paoline.

Croatto, J.S. (1985). L'herméneutique biblique en face des méthodes critiques: défi et perspectives. Vetus Testamentum, 36,77.

\section{(1987). Biblical Hermeneutics.} Nueva York: Orbis Books.

Dumont, J. (1990). La Praxeología ¿Qué ciencia para qué prácticas en el campo de la información? Association Francophone Internationale de Recherche Scientifique en Education. Recuperado de http://www. anuies.mx/servicios/p_anuies/publicaciones/revsup/res085/txt4.htm.

Eichrodt, W. (1975). Teología del Antiguo Testamento, v. I-II. Madrid: Cristiandad.
Espinas, A. (1897). Les origines de la technologie. París: Alcan.

Goppelt, L. (1982). Teologia del Nuovo Testamento. Brescia: Morcelliana.

Juliao, C. (2011). El enfoque praxeológico. Bogotá: Uniminuto.

Kraus, H. (1979). La teologia biblica, storia e problematica. Brescia: Paideia Editrice

Lemke, W. E. (1992). Old testament theology. En D. Freedman (Ed.), The anchor bible dictionary, v. Vi (pp. 448-472). New York: Doubleday.

Levorati, A. J. (1977). Lenguaje y Hermenéutica. Revista Bíblica, 39, 291-396.

Lodz, (1955). Traite du bon travail. Polonia: Lodz.

Morgan, R. (1992). New testament theology. En D. Fredman (Ed.), The anchor bible dictionary, v. vi (pp. 473-483). New York: Doubleday.

Parmentier, E. (2004). L'Ecriture vive, interprétations chrétiennes de la Bible, Géneve: Labor et Fides.

Pontificia Comisión Bíblica. (1993). La Interpretación de la Biblia en la Iglesia. Ciudad del Vaticano: Editrice Vaticana. 
Ramos Pérez, F. (2004). Ver a Jesús y sus signos, y creer en Él. Roma: AnGr 292.

Sánchez, W. G. (2009). La voz como modo de revelación. Roma: Editrice Pontificia Università Gregoriana.

Segalla, G. (1994). Panoramas del Nuevo Testament. Estella: Verbo divino.

(2006). Canone biblico e teologia biblica. un rapporto necesario... difficile. Liber annus, 56, 179-212.

Simian-Yofre, H. (2001). Metodología del Antiguo Testamento. Salamanca: Sígueme.
Schensohn, D. (2011). El poder de la acción a través de comunicación estratégica. Buenos Aires: Granica.

SluckiI, E. (1926). Ein Beitrag zur formal Praxeologischen Grundlegung derÖkonomik. Kiev: Universidad de Kiev.

Von Mises, L. (1983). L'action humaine; traité d'économie. Paris: PUF.

Von Rad, G. (1982). Teología del Antiguo Testamento, v. I-II. Salamanca: Sígueme.

Walter. (1975). Teología del Antiguo Testamento, v. I-II. Madrid: Cristiandad. 